\title{
ECONOMIC EVALUATION STUDIES OF SELF-MANAGEMENT INTERVENTIONS IN CHRONIC DISEASES: A SYSTEMATIC REVIEW
}

Mitchel van Eeden

CAPHRI, School for Public Health and Primary Care, Department of Health Services Research, Faculty of Health, Medicine and Life Sciences; Maastricht University

Department of Psychiatry \& Neuropsychology, Faculty of Health, Medicine \& Life Sciences MHeNS, School for Mental Health \& Neuroscience; Maastricht University

mitchel.vaneeden@maastrichtuniversity.nl

\section{Caroline M. van Heugten}

Department of Psychiatry \& Neuropsychology, Faculty of Health, Medicine \& Life Sciences MHeNS, School for Mental Health \& Neuroscience; Maastricht University
Department of Neuropsychology \& Psychopharmacology, Faculty of Psychology \& Neuroscience, Maastricht University

Ghislaine A.P.G. van Mastrigt

CAPHRI, School for Public Health and Primary Care, Department of Health Services Research, Faculty of Health, Medicine and Life Sciences; Maastricht University

Silvia M.A.A. Evers

CAPHRI, School for Public Health and Primary Care, Department of Health Services Research, Faculty of Health, Medicine and Life Sciences; Maastricht University

Trimbos Institute, Netherlands Institute of Mental Health and Addiction, Department of Public Mental Healthcare, Utrecht

Background: To our knowledge, there has been no overall systematic review focusing on the methodological quality of full economic evaluation studies of self-management interventions. Our objective was to systematically review the literature of full economic evaluation studies of self-management interventions in adult chronic patients and to investigate their methodological quality and cost-effectiveness.

Methods: A data extraction form was developed to assess general and randomized controlled trial (RCT) -related characteristics, quality, of the RCTs, economic information and quality of the economic evaluation studies by means of a quality assessment (CHEC-list for trial-based studies, adjusted CHEC-list for model-based studies).

Results: Twenty-three reports were found. Sixteen studies (73 percent) lack information on the control intervention(s). Only one study fulfilled all three criteria for quality of RCTs and five studies (23 percent) did not meet any of these criteria. This review included one model-based study; the other studies were trial-based economic evaluation studies based on a RCT. Eight studies (35 percent) used a societal perspective and 12 (60 percent) synthesized costs and effects. Seven studies were categorized into the highest category ( $<15$ score), nine studies into the "moderate" group (9-14 score), six studies received a "low" score $(<8)$ on the CHEC-list. Eighteen studies found the self-management intervention(s) to be cost-effective compared with other interventions

Conclusions: Self-management interventions for adult chronic patients were heterogeneous and there was no clear, well-considered definition of self-management. Overall, the methodological quality of the full economic evaluation studies was moderate and, therefore, cost-effectiveness results must be interpreted with caution. Future research will benefit from further improvements in methodological quality of both economic study design and analysis, as well as a taxonomy for describing self-management interventions and their contents.

Keywords: Systematic review, Economic evaluation, Self-management, Chronic health care

According to the World Health Organization (WHO), chronic diseases are defined as diseases of long duration and generally slow progression (1). Longer life expectancy and increasing numbers of people living with chronic diseases go along with

The authors appreciate the language review of this paper provided by Barbara Greenberg. This systematic review is embedded in the Restore4Stroke research program. Restore4Stroke is coordinated by ZonMw (Dutch Organization for Health Research and Development) and funded through the VSBfonds (the Dutch organization for supporting Dutch society with money, knowledge, and networks). Author Contributions: M.v.E. performed the database search and screening for articles. M.v.E., C.v.H., S.E., and G.v.M. participated in reviewing and scoring the articles included for this review. M.v.E. wrote the initial draft of the manuscript. C.v.H., S.E., and G.v.M. participated in the drafting of the manuscript and helped with the discussion of the manuscript. All authors read and approved the final manuscript. the greater proportion of elderly people in Western society (2). In parallel, healthcare costs are increasing in Western countries. The cost of chronic diseases and their risk factors are significant and can rise to almost 7 percent of a country's gross domestic product (GDP) (3). Governments face the challenge to meet, on the one hand, all of society's healthcare related needs and demands, and, on the other hand, the need to deal with scarce financial and material healthcare resources. The increased availability of new, often more expensive, healthcare interventions for chronic patients makes it imperative for policy makers to make a rational choice between alternatives.

Self-management is one of the healthcare interventions, which has recently become more prominent and important in chronic health care as it focuses on reaching optimization, 
efficiency, and sustainability of care by managing the existing intervention. In 1999, Lorig et al. (4) developed the Stanford Chronic Disease Self-Management Program (CDSMP). The effectiveness of the CDSMP, based on Bandura's Social Learning Theory (5), was recently investigated in a systematic review conducted by Franek (6). He found that, in comparison with care as usual, CDSMP resulted in significant, short term improvements in terms of health related quality of life and self-effectiveness. The popularity of this particular intervention has risen over the past two decades.

Defining self-management is a challenge, because a wide variety of interpretations and explanations exist. Self-management is often used interchangeably with self-care, self-help, and education, among others. Barlow et al. (2) defined self-management as: "An individual's ability to manage the symptoms, treatment, physical, and psychosocial consequences and lifestyle changes inherent in living with a chronic condition. Efficacious self-management encompasses the ability to monitor one's condition and to affect the cognitive, behavioral, and emotional responses necessary for maintaining a satisfactory quality of life and, thus, establish a dynamic and continuous process of self-regulation" (2). Because this definition adequately captures the domain of self-management, we choose to use this definition in this review and articles are selected in line with this definition.

There are four reasons for doing this review. First, the significance of self-management interventions (SMIs) in chronic health care and pharmaceutical therapy has been acknowledged $(7 ; 8)$. Second, previous research shows positive results regarding the effectiveness of SMIs (9). Third, there is potential for SMIs to be cost-effective for specific chronic diseases. However, current evidence on the cost-effectiveness of SMIs is limited. Fourth, following the previous arguments, this calls for new, up-to-date evidence concerning the methodological quality of these studies and the effect of this quality on the interpretation of cost-effectiveness results.

To our knowledge, there has been no overall systematic review focusing on the methodological quality of full economic evaluation studies of SMIs. Therefore, our aim was to systematically review the literature of full economic evaluation studies, both trial-based and model-based, of one or more SMIs in adult chronic patients to investigate the methodological quality of the studies and the cost-effectiveness of the interventions.

\section{METHODS}

\section{Study Approach}

This review is conducted according to the PRISMA guidelines, containing twenty-seven items. This study was not registered in the international prospective register of systematic reviews (PROSPERO) before starting but we used a predefined research protocol.

\section{Selection Criteria}

Full economic evaluation studies reporting on SMIs in line with Barlow's definition (2) of self-management were eligible for inclusion. A full economic evaluation is considered a comparative analysis of two or more interventions in terms of both costs (resource use) and consequences (outcomes, effects) (10). It was our goal to include as many relevant papers as possible, however, we focused on studies reporting a chronic disease. Studies were excluded if patients could not be classified as having a chronic disease or receiving chronic care, if participants were younger than 18 years of age, if the study was not written in English or Dutch and/or was published before 1990. A chronic patient was defined as a person who experiences living with the affliction that often accompanies chronic disease (11). Furthermore, we excluded studies when they did not report on original data in a primary research paper (i.e., systematic reviews, congress abstracts, and commentaries, among others).

\section{Information Sources}

Two electronic databases were searched for relevant articles published between January 1990 and May 2014. Because the majority of SMIs was developed and studied for their efficacy in the past decade, we chose 1990 as the starting point for our search. We used PUBMED and the National Institute for Health Research Economic Evaluation Database (NHSEED). Although NHSEED makes an extensive search of PUBMED among others (CINAHL, Embase, PsycINFO, and MEDLINE) on a weekly basis, using a very efficient filter embedded in their search engine for economic evaluation studies, we performed a separate search in PUBMED as well as this combination is regarded as the most valid search strategy for economic evaluation studies $(12 ; 13)$. In addition, reference lists were screened for additional relevant publications.

\section{Search Strategy}

The search string used in this study was based on leading reviews of self-management and existing economic evaluation search strategies $(2 ; 12-16)$. The following set of keywords was used to search PUBMED, either as a MeSH term if possible, or as general search term title/abstract: ["self management"], ["selfmanagement"], ["self care"], ["self-care"]; and ["economics"], ["cost analysis"], ["cost benefit analysis"], ["cost-benefit analysis"], ["cost effective"], ["cost-effective"]. Because NHSEED searches only databases for economic evaluation studies, only the set of keywords for self-management was used to identify relevant studies in this database. We used a "language" filter (either Dutch or English) and searched only for articles published in or after 1990. All results were downloaded into the bibliographic management software EndNoteX and duplicates were removed. A detailed search string is presented in Supplementary Figure 1. 
van Eeden et al.

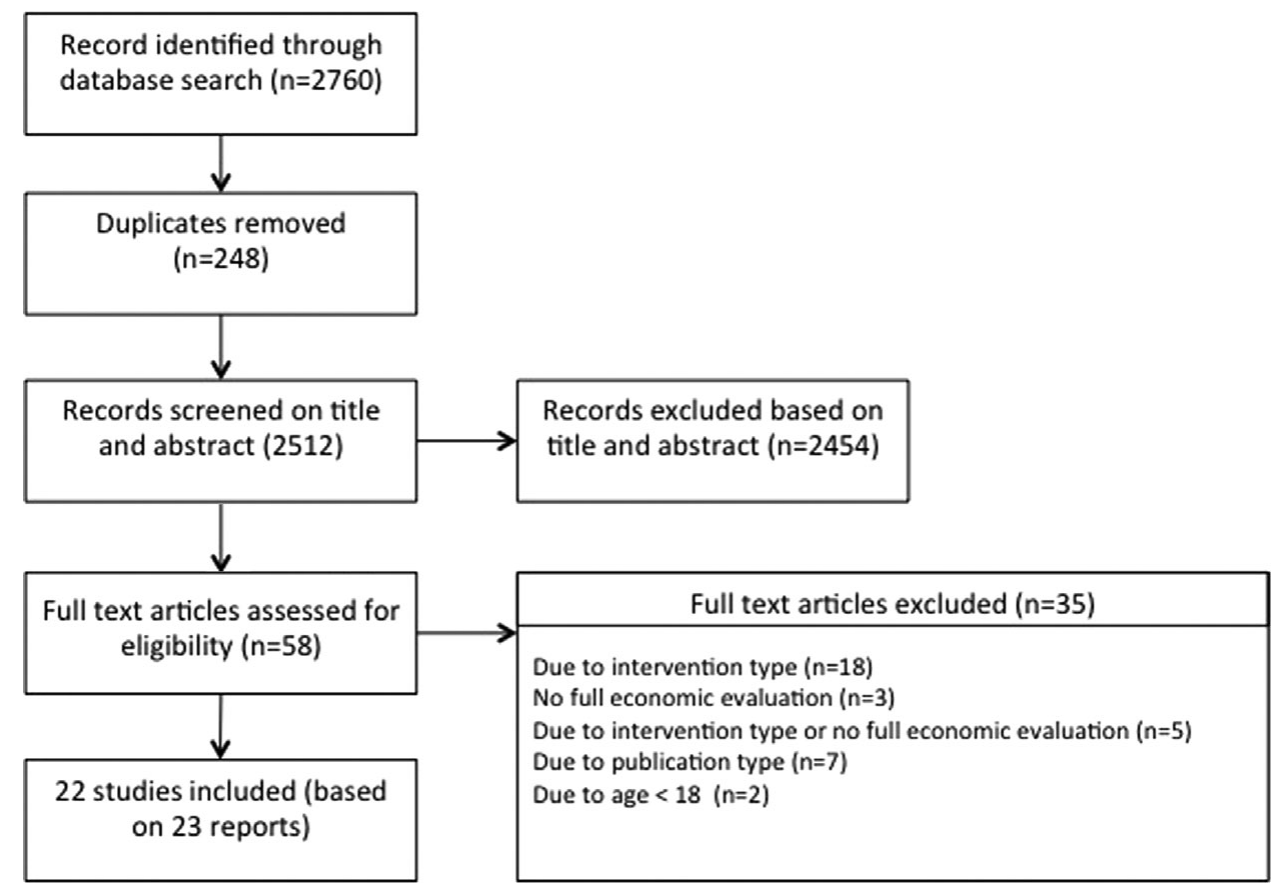

Figure 1. Flowchart of study selection.

\section{Study Selection}

Eligibility assessment was performed by the primary researcher (M.v.E.) and three other reviewers (C.v.H., S.E., and G.v.M.). M.v.E. did the electronic database search and screened all articles on title and abstract. All papers that met the eligibility criteria were categorized in the group as "potential," and papers that undoubtedly did not meet these criteria were immediately excluded. In case of doubt, decisions were made through consensus by two of the four reviewers (M.v.E. with C.v.H., and G.v.M. with S.E.). Subsequently, all articles meeting the eligibility criteria were assessed independently by two of the four reviewers. This assessment consisted of data extraction of all selected full-text articles in addition with a critical appraisal of the Consensus Health Economic Criteria List (CHEC-list). Any disagreement on data extraction between reviewers was resolved through consensus.

\section{Data Extraction and Methodological Quality Assessment}

By means of a predefined form (17), the following data were extracted from the papers: First, general and randomized controlled trial (RCT) information was retrieved. Quality of RCTs was determined through assessment: the use of intention-totreat analysis (ITT) (yes, no, unclear), allocation concealment (adequate, unclear), and blinding of outcome assessors (adequate, unclear) (18). If the paper reporting on the economic evaluation study did not contain sufficient information on trial design, the paper describing the original study design was used for data extraction. Second, economic information was assessed, including the economic evaluation design used, synthesis of costs and effects, and cost-effectiveness of the SMI (yes/no).
The quality of the economic evaluation was determined by means of the adjusted CHEC-list which is fit for RCTs and model-based studies (19) consisting of nineteen yes-or-no questions (20). The CHEC-list is recommended by Cochrane (18). Because no weighting exists for the CHEC-list, we used scores of 1 (yes) and 0 (no) to value the items and categorized studies as: low ( $<8$ score), moderate (9-14 score) and high ( $>15$ score). For studies that did not discount costs and/or effects due to a 12-month or less follow-up, item 14 (were future costs and outcomes discounted) on the CHEC-list was disregarded and for these studies a maximum score of eighteen could be obtained. However, this had no impact on the categorization of studies.

\section{RESULTS}

\section{Review Profile}

A total of 2,760 records were identified from the search (Figure 1). After removing duplicates $(n=248), 2,512$ records were screened on title and abstract; of these, 2,454 records were excluded. The main reasons for exclusion were: not reporting data in a primary research paper, using a description of selfmanagement not in line with the Barlow's definition and the absence of cost data. A total of fifty-eight full text records were then assessed by the reviewers, of which twenty-three fulfilled the inclusion criteria (21-43). One study performed by Kauppinen et al. was published as an additional publication (29) with respect to a previous study (28). The data of this publication were also extracted and presented in this review, but for simplification purposes only the reference of the original article (28) 
will be used in the text. Accordingly, the results are based on twenty-three publications from twenty-two studies.

\section{General, RCT-Related and Economic Characteristics}

All twenty-two studies were published after 1998 (Table 1, Supplementary Tables 1 and 2), eight were based in the United Kingdom $(24 ; 27 ; 30 ; 31 ; 34 ; 36 ; 38 ; 40)$, six in mainland Europe: the Netherlands (33;35), Finland (28), Sweden (22), Norway (23), Spain (26), six in the United States (US) $(25 ; 37 ; 39 ; 41-$ 43) and two in Canada (21;32). Asthma/chronic obstructive pulmonary disease (COPD) and diabetes were the most common disease categories in eleven studies. One study used data from an RCT to construct a Markov model. All others used an RCT design including two, three, or four intervention groups. A total of 23 percent $(n=5)$ of the studies evaluated two or more SMIs.

The intensity of the SMI varied between studies, from two visits and a phone call up to sixty computer sessions per participant. The SMI was primarily delivered by a (trained) nurse in eleven studies. Almost one-third $(n=8)$ of the interventions were offered to patients at home. In three studies, interventions took place in the hospital, in three studies interventions were at a special clinic and in three other studies interventions were at the general practitioner's (GP) office. All SMIs consisted of multiple sessions (individual or group), sometimes combined with phone calls. Sixteen studies (73 percent) provided limited or missing information on the description of the control intervention(s), the other 27 percent of the studies were thorough in reporting on the control intervention(s). Only one study had a 10-week follow-up period. A 12-month follow-up period was chosen in fifteen studies, and the other seven studies had follow-up periods ranging from 18 months up to 10 years.

In seven studies ( 32 percent), ITT analyses were performed; in other words, all randomized patients were analyzed in the assigned group regardless of whether they completed the followup period or received the treatment (ITT). The remaining 15 studies either did not us an ITT analysis method $(n=8)$ or it was unclear whether an ITT analysis was conducted $(n=7)$. Twelve studies $(n=12)$ reported adequately concerning the concealment of allocation. Limited to no information on concealment of allocation was provided in the remaining ten studies. Only four studies (18 percent) reported blinding of the outcome assessor(s). The remaining eighteen studies either mentioned that a nonblinded study was concerned, or insufficient information was provided on blinding.

In 68 percent $(n=15)$ of the studies, a perspective of analysis was mentioned and almost half of these $(n=8)$ performed their analyses from a societal perspective. In 32 percent of the studies $(n=7)$, no information was provided on the chosen perspective. All but four of the studies performed either a costeffectiveness analysis (CEA) or cost utility analysis (CUA), although it is worth mentioning that authors used both concepts interchangeably. The four other studies conducted either a cost analysis or cost-minimization analysis (CMA). Two studies did not measure any type of healthcare costs (categorized as hospital visits, GP visits, and other healthcare consumption, among others).

Patient and family costs (categorized as costs of informal care and travel costs, among others) were not identified in ten studies. In eleven studies, no costs outside the healthcare sector (categorized as productivity costs, among others) were identified. One study took intervention costs into account. All but three of the studies used a cost questionnaire, medical/hospital records, or both, among other instruments as means for measuring costs. Approximately 55 percent of the studies $(n=12)$ based their cost valuation on either cost price estimations, cost guidelines, or both. The remaining 45 percent of the studies ( $n$ $=10$ ) used either tariffs, tariffs combined with cost guidelines, cost price estimations combined with the human capital approach, tariffs combined with nurse wages, or did not mention any form of cost valuation. Almost half of the studies $(n=10)$ did not mention a price year with respect to the cost calculation. Four of eight studies with a follow-up period of 18 months or more did not report a discount rate.

Ten studies used only generic effect measures, four used only disease specific effect measures and eight used both. In seven studies, uncertainty was handled using test variable uncertainty methods (e.g., sensitivity analysis), five studies used test sample uncertainty methods (e.g., bootstrapping) and in three studies a combination of these was used. Other methods of handling uncertainty were probabilistic analysis and test sample uncertainty combined with complete case analysis. In five studies, no handling of uncertainty was mentioned. Thirteen studies (59 percent) used an incremental cost-effectiveness ratio (ICER) to synthesize costs and effects, and one study combined the ICER with the calculation of a Net Monetary Benefit (NMB). The remaining nine studies did not mention any method for synthesizing costs and effects. Almost half of the studies $(n=10)$ made cost-effectiveness results visible. Seven studies presented a cost-effectiveness acceptability curve (CEAC), the remaining three used either a cost-effectiveness plane (CE plane), a CEAC and $\mathrm{CE}$ plane combined, or a cost-concentration curve.

\section{Quality of the Economic Evaluation}

A total of fourteen studies could obtain a maximum of 18 points on the CHEC-list (Table 2); the other eight could obtain nineteen points. Seven studies were categorized into the highest category ( $>15$ score $(25 ; 30 ; 31 ; 33 ; 35 ; 36 ; 38)$, nine studies into the group "moderate" (9-14 score) $(22-24 ; 27 ; 28 ; 37 ; 39 ; 42 ; 43)$, and six studies received a "low" score $(<8)(21 ; 26 ; 32 ; 34 ; 40 ; 41)$. On average, all studies included in this review scored 13.9 on the CHEC-list range 4-17. Studies published before $2007(n=10)$ had on average a lower score in comparison with the studies published in 2008 or later $(n=12)$. There were several CHEC-list 
Table 1. General, RCT-Related and Economic Characteristics

\begin{tabular}{|c|c|c|c|c|c|c|c|c|}
\hline Disease $^{a}$ & Author & N & $\begin{array}{l}\text { Self-management } \\
\text { Interventions }\end{array}$ & Comparator(s) & $\begin{array}{l}\text { Outcome } \\
\text { measure(s) }\end{array}$ & Perspective ${ }^{b}$ & $\begin{array}{l}\text { Cost } \\
\text { measurement }\end{array}$ & $\begin{array}{l}\text { SMl cost- } \\
\text { effective }\end{array}$ \\
\hline \multirow[t]{4}{*}{ Asthma } & $\begin{array}{r}\text { Gallefos }^{23} \\
(2001)\end{array}$ & 78 & $\begin{array}{l}\text { SMIC with group/individual sessions provided by a } \\
\text { trained nurse of physiotherapist, following an } \\
\text { individual SM plan }\end{array}$ & - Usual care & $\begin{array}{l}\text { - Qo } L^{d} \\
\text { - Costs } \\
\text { - Forced expiratory } \\
\text { volume }\end{array}$ & - Society & $\begin{array}{l}\text { - Medical } \\
\text { records } \\
\text { - Registrations }\end{array}$ & Yes \\
\hline & $\begin{array}{l}\text { Kauppinen }{ }^{28} \\
\text { (1998) }\end{array}$ & 162 & $\begin{array}{l}\text { SM plan to manage individual asthma peak-flow } \\
\text { meters and record values in a special diary }\end{array}$ & $\begin{array}{l}\text { - Educational } \\
\text { program }\end{array}$ & $\begin{array}{l}\text { - QoL } \\
\text { - Costs } \\
\text { - Forced expiratory } \\
\text { volume }\end{array}$ & $\mathrm{Nl}$ & $\begin{array}{l}\text { - Medical } \\
\text { records }\end{array}$ & Yes \\
\hline & $\begin{array}{r}\text { McLean }^{32} \\
(2003)\end{array}$ & 242 & $\begin{array}{l}\text { Enhanced pharmaceutical care including teach of } \\
\text { self-management according to the HOP Asthma } \\
\text { Care Module }\end{array}$ & - Usual care & $\begin{array}{l}\text { - QoL } \\
\text { - Costs } \\
\text { - Peak flow }\end{array}$ & $\mathrm{Nl}$ & $\begin{array}{l}\text { - Medical } \\
\text { records }\end{array}$ & Yes \\
\hline & $\begin{array}{l}\text { Van der } \\
\text { Meer }^{33} \\
(2010)\end{array}$ & 200 & $\begin{array}{l}\text { Internet based SMI including online/group-based } \\
\text { education according to a computerized personal } \\
\text { action plan }\end{array}$ & - Usual care & $\begin{array}{l}\text { - Qol } \\
\text { - Costs }\end{array}$ & - Society & $-C Q^{e}$ & Yes \\
\hline & $\begin{array}{r}\text { Handley }{ }^{25} \\
(2008)\end{array}$ & 226 & $\begin{array}{l}\text { Automated telephoned SMI providing surveillance } \\
\text { and education including nurse care management } \\
\text { and one-on-one telephone counselling }\end{array}$ & - Usual care & $\begin{array}{l}- \text { QoL } \\
\text { - Costs }\end{array}$ & $\begin{array}{l}\text { - Health } \\
\text { systems }\end{array}$ & $\begin{array}{l}\text { - Intervention } \\
\text { costs }\end{array}$ & Yes \\
\hline & $\begin{array}{l}\operatorname{Jansa}^{26} \\
(2006)\end{array}$ & 40 & $\begin{array}{l}\text { Telecare SMI focusing on informed decision making } \\
\text { about daily diabetes self-management }\end{array}$ & - Usual care & $\begin{array}{l}\text { - QoL } \\
\text { - Costs } \\
\text { - Metabolic control }\end{array}$ & $\mathrm{Nl}$ & $-C Q$ & Yes \\
\hline & $\begin{array}{l}\text { Ritzwoller }^{39} \\
(2011)\end{array}$ & 280 & $\begin{array}{l}\text { Viva Bien SMI based on social-cognitive, } \\
\text { social-ecologic, and problem-solving factors to } \\
\text { improve risk factors known to affect coronary } \\
\text { heart disease }\end{array}$ & - Usual care & $\begin{array}{l}- \text { QoL } \\
\text { - Costs }\end{array}$ & $\begin{array}{l}\text { - Clients and } \\
\text { health plan }\end{array}$ & $\begin{array}{l}-C Q \\
- \text { Intervention } \\
\text { logs }\end{array}$ & Yes \\
\hline
\end{tabular}


Table 1. Continued

\begin{tabular}{|c|c|c|c|c|c|c|c|c|}
\hline Disease $^{a}$ & Author & $\mathrm{N}$ & $\begin{array}{l}\text { Self-management } \\
\text { Interventions }\end{array}$ & Comparator(s) & $\begin{array}{l}\text { Outcome } \\
\text { measure(s) }\end{array}$ & Perspective ${ }^{b}$ & $\begin{array}{l}\text { Cost } \\
\text { measurement }\end{array}$ & $\begin{array}{l}\text { SMI cost- } \\
\text { effective }\end{array}$ \\
\hline \multirow[t]{5}{*}{$\begin{array}{l}\text { Cardiovascular } \\
\text { disease }\end{array}$} & $\begin{array}{l}\text { Cline }^{22} \\
\quad(1998)\end{array}$ & 199 & $\begin{array}{l}\text { SM education program including guidelines for } \\
\text { self-management of diuretics and providing } \\
\text { information and individual guidelines }\end{array}$ & - Usual care & $\begin{array}{l}\text { - Hospitalization } \\
\text { - Re-admission time } \\
\text { - Costs }\end{array}$ & $\mathrm{Nl}$ & $\begin{array}{r}\text { - Hospital } \\
\text { records }\end{array}$ & Yes \\
\hline & $\begin{array}{l}\text { Jowett } \\
\text { (2006) }\end{array}$ & 617 & $\begin{array}{l}\text { SM anticoagulation program providing training and } \\
\text { self-management tools to manage individual } \\
\text { anticoagulation }\end{array}$ & - Usual care & $\begin{array}{l}- \text { QoL } \\
\text { - Costs }\end{array}$ & - Society & $\begin{array}{l}-C Q \\
- \text { Medical } \\
\text { records }\end{array}$ & No \\
\hline & $\begin{array}{l}\text { Reed }^{37} \\
\quad(2010)\end{array}$ & 636 & $\begin{array}{l}\text { Tailored SMI incorporating behavioral and } \\
\text { educational aspects of treatment adherence }\end{array}$ & $\begin{array}{l}\text { - Blood pressure } \\
\text { monitoring } \\
\text { - Usual care }\end{array}$ & $\begin{array}{l}\text { - Patient time } \\
\text { - Costs }\end{array}$ & - Society & $\begin{array}{l}-C Q \\
\text { - Telephone } \\
\text { calls }\end{array}$ & Yes \\
\hline & $\begin{array}{c}\text { Bourbeau }^{21} \\
(2006)\end{array}$ & 191 & $\begin{array}{l}\text { SMI on education including teaching at home and } \\
\text { telephone calls by a case manager }\end{array}$ & - Usual care & $\begin{array}{l}\text { - Hospitalization } \\
\text { - Costs }\end{array}$ & $\begin{array}{l}\text { - Healthcare } \\
\text { payer }\end{array}$ & $\begin{array}{l}\text { - Interviews } \\
\text { - Hospital } \\
\text { records }\end{array}$ & Yes \\
\hline & $\begin{array}{l}\text { Khdour }^{30} \\
(2011)\end{array}$ & 127 & $\begin{array}{l}\text { Pharmacy-led SMI education program to increase } \\
\text { patients' self-efficacy and manage breathing } \\
\text { difficulties }\end{array}$ & - Usual care & $\begin{array}{l}- \text { QoL } \\
- \text { Costs }\end{array}$ & $\begin{array}{l}\text { - NHS } \\
\text { - Personal } \\
\text { social service }\end{array}$ & $\begin{array}{l}-C Q \\
- \text { Medical } \\
\text { records }\end{array}$ & Yes \\
\hline \multirow[t]{2}{*}{$\begin{array}{l}\text { Neurological } \\
\text { problems }\end{array}$} & $\begin{array}{l}\text { Latimer }^{31} \\
\quad(2013)\end{array}$ & 34 & $\begin{array}{l}\text { Self-managed computer therapy with speech and } \\
\text { language therapy provided by a volunteer }\end{array}$ & - Usual care & $\begin{array}{l}- \text { QoL } \\
\text { - Costs }\end{array}$ & $\begin{array}{l}\text { - Healthcare } \\
\text { sector } \\
\text { - Personal } \\
\text { social service }\end{array}$ & $\begin{array}{l}\text { - Patient and } \\
\text { carer diaries }\end{array}$ & Yes \\
\hline & $\begin{array}{l}\text { Moss- } \\
\text { Morris } \\
(2012)\end{array}$ & 45 & $\begin{array}{l}\text { Internet-based cognitive behavioral SMI including } \\
\text { computer sessions and telephone calls }\end{array}$ & - Usual care & $\begin{array}{l}\text { - Fatigue } \\
\text { - QoL } \\
\text { - Costs }\end{array}$ & $\mathrm{NI}$ & $-C Q$ & Yes \\
\hline
\end{tabular}

기 
Table 1. Continued

\begin{tabular}{|c|c|c|c|c|c|c|c|c|}
\hline Disease $^{a}$ & Author & N & $\begin{array}{l}\text { Self-management } \\
\text { Interventions }\end{array}$ & Comparator(s) & $\begin{array}{l}\text { Outcome } \\
\text { measure(s) }\end{array}$ & Perspective ${ }^{b}$ & $\begin{array}{l}\text { Cost } \\
\text { measurement }\end{array}$ & $\begin{array}{l}\text { SMl cost- } \\
\text { effective }\end{array}$ \\
\hline \multirow[t]{5}{*}{ Other } & $\begin{array}{l}\text { Van 0s- } \\
\text { Medendorp }{ }^{35} \\
\text { (2012) }\end{array}$ & 199 & $\begin{array}{l}\text { Individualized e-health SMI incorporating } \\
\text { self-management training and internet-guided } \\
\text { monitoring, using an internet portal }\end{array}$ & - Usual care & $\begin{array}{l}\text { - Atopic dermatitis } \\
\text { severity } \\
\text { - QoL } \\
\text { - Costs }\end{array}$ & - Society & $\begin{array}{l}-C Q \\
- \text { Records } \\
\text { - Diaries }\end{array}$ & Yes \\
\hline & $\begin{array}{l}\text { Patel }^{36} \\
\text { (2009) }\end{array}$ & 812 & $\begin{array}{l}\text { SM program plus education booklet (Challenging } \\
\text { Arthritis) designed to improve self-efficacy, } \\
\text { based on social cognitive theories }\end{array}$ & - Education & $\begin{array}{l}- \text { QoL } \\
\text {-Costs }\end{array}$ & - Society & $\begin{array}{l}-C Q \\
\text { - Client service } \\
\text { receipt }\end{array}$ & No \\
\hline & $\begin{array}{l}\text { Richardson }{ }^{38} \\
\text { (2013) }\end{array}$ & 296 & $\begin{array}{l}\text { Pragmatic rehabilitation self-management } \\
\text { program: an individualized program of activity } \\
\text { and improved sleep }\end{array}$ & $\begin{array}{l}\text { - Supportive } \\
\text { listening } \\
\text { - Usual care }\end{array}$ & $\begin{array}{l}- \text { Qol } \\
\text { - Costs }\end{array}$ & $\begin{array}{l}\text { - NHS } \\
\text { - Social } \\
\text { service } \\
\text { perspec- } \\
\text { tive }\end{array}$ & $-C Q$ & No \\
\hline & $\begin{array}{l}\text { Robinson }{ }^{40} \\
(2005)\end{array}$ & 420 & $\begin{array}{l}\text { Self-help group meetings to share experiences of } \\
\text { living with functional bowel symptoms and } \\
\text { providing approaches to help manage symptoms }\end{array}$ & - Guidebook & $\begin{array}{l}\text { - Consultations } \\
\text { - Costs }\end{array}$ & $\mathrm{NI}$ & $\begin{array}{l}-C Q \\
\text { - Primary care } \\
\quad \text { records }\end{array}$ & Yes \\
\hline & $\begin{array}{l}\text { Wang }{ }^{43} \\
(2012)\end{array}$ & 591 & $\begin{array}{l}\text { Telephone-based SMI providing individual training } \\
\text { and a blood-pressure device, in combination with } \\
\text { behavioral treatment }\end{array}$ & $\begin{array}{l}\text { - Medication } \\
\text { management } \\
\text { - Usual care }\end{array}$ & $\begin{array}{l}\text { - Blood pressure } \\
\text { - QoL } \\
\text { - Costs }\end{array}$ & $\begin{array}{l}\text { - Healthcare } \\
\text { sector }\end{array}$ & $\begin{array}{r}\text { - Hospital } \\
\text { records }\end{array}$ & No \\
\hline
\end{tabular}

"Disease categories are further explained in Supplementary Table 1.

${ }^{b} \mathrm{NI}, \mathrm{no}$, or limited, information available.

CSMI, self-management intervention.

${ }^{\mathrm{d}} \mathrm{Q} \mathrm{L}$, quality of life.

${ }^{e} C Q$, cost questionnaire.

NNHS, National Health Service. 
Table 2. Critical Appraisal of the Quality of the Economic Evaluation ( $+=$ the Article Sufficiently Handled the CHEC-List Criterion)

\begin{tabular}{|c|c|c|c|c|c|c|c|c|c|c|c|c|c|c|c|c|c|c|c|}
\hline & $\begin{array}{c}\text { Study } \\
\text { population } \\
\text { described }\end{array}$ & $\begin{array}{c}\text { Alter- } \\
\text { natives } \\
\text { described }\end{array}$ & $\begin{array}{l}\text { Research } \\
\text { question }\end{array}$ & $\begin{array}{l}\text { Appr. } \\
\text { design }\end{array}$ & $\begin{array}{c}\text { Appr. } \\
\text { Time } \\
\text { horizon }\end{array}$ & $\begin{array}{l}\text { Persp- } \\
\text { ective }\end{array}$ & $\begin{array}{l}\text { Relevant } \\
\text { costs }\end{array}$ & $\begin{array}{l}\text { Costs in } \\
\text { physical } \\
\text { units }\end{array}$ & $\begin{array}{l}\text { Costs } \\
\text { valued } \\
\text { appr. }\end{array}$ & $\begin{array}{l}\text { Relevant } \\
\text { effects }\end{array}$ & $\begin{array}{l}\text { Effects } \\
\text { in } \\
\text { physical } \\
\text { units }\end{array}$ & $\begin{array}{l}\text { Effects } \\
\text { valued } \\
\text { appr. }\end{array}$ & $\begin{array}{c}\text { Incre- } \\
\text { mental } \\
\text { analysis }\end{array}$ & $\begin{array}{c}\text { Dis- } \\
\text { counting }\end{array}$ & $\begin{array}{l}\text { Sensitivity } \\
\text { Analysis }\end{array}$ & $\begin{array}{l}\text { Conclusions } \\
\text { follow } \\
\text { data } \\
\text { reported }\end{array}$ & $\begin{array}{l}\text { Genera- } \\
\text { lizability }\end{array}$ & $\begin{array}{l}\text { No } \\
\text { conflict } \\
\text { of } \\
\text { interest }\end{array}$ & $\begin{array}{l}\text { Ethical } \\
\text { issues }\end{array}$ \\
\hline Gallefos ${ }^{23}$ & + & + & & + & + & + & + & + & + & + & + & + & + & $*$ & + & + & + & & \\
\hline Kauppinen ${ }^{28}$ & + & & + & + & + & & & & + & + & + & & + & + & & + & & & \\
\hline Mclean $^{32}$ & & & & & + & & & & & + & + & & & $*$ & & + & & & \\
\hline Van der $\mathrm{Meer}^{33}$ & + & + & & + & + & + & + & + & + & + & + & + & + & $*$ & + & + & & + & + \\
\hline Schermer ${ }^{41}$ & + & & + & + & + & + & + & & + & & + & & & & & & & & \\
\hline Gillet24 & & & & + & + & & + & + & + & + & + & + & + & + & + & + & & + & + \\
\hline Handley 25 & + & + & + & + & + & + & + & + & + & & + & + & + & $*$ & + & + & + & + & + \\
\hline Jansa 26 & + & & & & + & & + & & & + & + & & & $*$ & & & & & \\
\hline Ritzwoller ${ }^{39}$ & + & + & + & & & & & + & + & + & + & + & & $*$ & + & + & + & + & + \\
\hline Cline 22 & + & + & + & + & + & & & + & + & + & + & + & & $*$ & + & + & + & + & + \\
\hline Jowett ${ }^{27}$ & & & & + & + & + & + & + & + & + & + & + & + & $*$ & + & + & & + & \\
\hline $\operatorname{Reed}^{37}$ & + & & & & + & + & & + & + & & & & & + & + & + & + & & \\
\hline Bourbeau $^{21}$ & + & & & & + & & + & & & & & & + & $*$ & & + & + & & + \\
\hline Khdour $^{30}$ & + & & + & + & + & & + & + & + & + & + & + & + & $*$ & + & + & + & + & \\
\hline Latimer $^{31}$ & + & & + & + & + & + & + & + & + & + & + & + & + & & + & + & & + & + \\
\hline Moss-Morris ${ }^{34}$ & + & & + & & & & & + & + & + & + & + & & $*$ & & & & + & \\
\hline Van Os-Medendorpp 35 & + & + & + & & + & + & & + & + & + & + & + & + & & & + & + & + & + \\
\hline Patel ${ }^{36}$ & + & + & + & + & + & + & + & + & + & + & + & + & + & $*$ & + & + & + & + & \\
\hline Richardson ${ }^{38}$ & + & + & + & + & + & + & & + & + & + & + & + & + & + & & + & + & + & + \\
\hline Robinson ${ }^{40}$ & + & + & & & + & & & & & & & & & $*$ & & & & + & \\
\hline Strong ${ }^{42}$ & & & & + & + & & + & + & & & + & & + & $*$ & + & + & & + & \\
\hline Wang ${ }^{43}$ & + & + & + & & + & + & + & + & & & + & + & & & + & + & + & & + \\
\hline
\end{tabular}

*Discounting not necessary due to follow-up up to 12 months. 
items on which various studies did not score points. For instance, twelve studies did not justify their choice of perspective. Half of the studies $(n=11)$ did not give a full identification of all relevant costs in relation to the perspective. Twelve studies did not give a detailed description of the competing interventions and ten studies did not perform a sensitivity analysis. Thirteen studies reported no ethical aspects and did not elaborate on the characteristics of the population experiencing the disease and intervention and how this may have distributional implications.

\section{Cost-Effectiveness}

In eighteen studies ( 82 percent), the authors found the SMI to be cost-effective (Table 3 ), either due to increased costs and effects in comparison with the control intervention $(24 ; 25 ; 33 ; 37 ; 42)$, or decreased costs and increased effects in comparison with the control intervention (21-23;26;28;30-32;34;35;39-41). One of the twenty-two studies did not find positive cost-effectiveness results at the 12 months follow-up, but in the additional publication focusing on long term cost-effectiveness the SMI proved cost-effective. Four studies concluded that the SMI was not costeffective; three found no significant differences in comparison with the control intervention and in one study the control intervention was cheaper and more effective and, therefore, dominant over the SMI.

\section{DISCUSSION}

As far as we know, this is the first overall systematic review of the methodological quality and cost effectiveness of full economic evaluation studies of SMIs in adult chronic patients. Twentytwo studies of twenty-three publications were identified, in most cases published either in Europe or the United States. All studies were published from 1998 onward and more than half of the studies were published after 2006. This review included one model-based and 21 trial-based economic evaluations. Most of the studies performed either a cost-effectiveness analysis or a cost-utility analysis.

The reported SMIs were very heterogeneous in terms of time span, intensity, contents, interventions providers, and target populations. In general, the methodological quality was moderate, despite the fact that the majority of studies were RCTs. Nevertheless, only one study fulfilled all three evaluated quality criteria (ITT, allocation concealment, and blinding of outcome assessor), and in five studies, none of these criteria were met. Furthermore, there was lack of detailed information on the control intervention(s) among all studies and in some cases nothing was reported.

Only seven of the twenty-two studies that reported data on costs had an acceptable score on the CHEC-list and the majority of studies had either a moderate or low score. The methodological quality of the economic evaluation was very mixed, but this was insufficient in a greater part of the studies, which were unclear on synthesizing costs and effects and sensitivity analyses.
Eleven studies did not report on the perspective of analysis but the majority of those who did used a societal perspective. However, the majority of studies with high methodological quality did show positive cost-effectiveness results of the SMIs. Also, these high quality studies were more likely to report a QALY as outcome measure compared with studies with moderate or even low methodological quality. Studies with higher methodological quality reported extensively on methods of analysis, and provided arguments for choices and considerations with regard to cost-analyses. We found that more recently published studies showed higher methodological quality. This might also be due to the fact that the economic evaluations were conducted as independent study and not integrated with the effect study.

We encountered several difficulties in selecting of studies reporting SMIs and in the categorization of these interventions. It was evident in advance that the absence of a clear and solid definition would pose difficulties. We chose a definition formulated by Barlow et al. (2) that in our view captured the full domain of self-management adequately and we used this definition as indicator for the selection of relevant articles for this review. Despite choosing this definition we found it a major challenge to use the definition in our research. This can be illustrated by the rejection of an article with self-management in its title as we found that the contents of the article and the description of self-management did not fit our definition (44).

Another difficulty was in categorizing SMIs. Selfmanagement can serve as an intervention on its own with, for example, educational sessions and self-help activities as sub-elements, or self-management can be a sub-element of an educational intervention. Furthermore, self-management, education, self-care, and self-help are continuously being used interchangeably, sometimes even for the same activities or programs in one study. A review conducted by Nolte and Osborne (45) on the outcomes of chronic disease self-management interventions found that due to the different types of SMIs it is very difficult to estimate the true impact of these interventions. These findings are indicative of the highly ambiguous and complex character of an intervention becoming vastly more popular among scientists and healthcare providers and the difficulties, which researchers encounter when reviewing the literature on this topic.

The majority of studies $(n=18)$ found the SMI(s) to be cost-effective in comparison with other, mostly care as usual, interventions. SMIs are usually multi-faceted programs in which different strategies and techniques are used and applied to help patients to manage their disease over time. Furthermore, SMIs tend to increase understanding and awareness among patients considering their own disease. This allows them to, for example, get a better idea of which healthcare services are necessary to cope with their current situation and may improve medicine adherence. This could easily result in a decrease of healthcare consumption and, therefore, a decrease in costs; hence, it seems plausible for self-management interventions to prove to be 
Table 3. Cost-Effectiveness Results

\begin{tabular}{|c|c|c|c|}
\hline Disease & Author & Main outcome $^{a}$ & ICER $^{a}$ \\
\hline \multirow[t]{20}{*}{ Asthma } & Gallefos ${ }^{23}(2001)$ & - Cost savings of 3400 NOK and 4500 & - -3,400 NOK per 10 point improvement on SGRQ \\
\hline & & - NOK per patient & $--4,500$ NOK per $5 \%$ improvement in FEV \\
\hline & & $\begin{array}{l}-10 \text {-unit improvement SGRQ } \\
-5 \% \text { REV }\end{array}$ & \\
\hline & Kauppinen ${ }^{28}$ (1998) & - SMI is more costly than the control intervention at & - 203 FIM (15D) \\
\hline & & 12 months & - 162 FIM (SGRQ) \\
\hline & & - Dominant control intervention at 12 months & - 140 FIM (FVC) \\
\hline & & - Negative net benefit at 12 months & -86 FIM (FEV) \\
\hline & & - 364 less sickness days for patients in self- & - 1,845 FIM (PD) \\
\hline & & management group at 5 years & - 110 FIM (PEF) \\
\hline & & $\begin{array}{l}\text { - No significant differences between groups in } \\
\text { clinical and health-related outcomes at } 5 \text { years }\end{array}$ & \\
\hline & McLean $^{32}$ (2003) & - Cost savings of 201 USD per patient & $\mathrm{NI}$ \\
\hline & & - Symptom scores decreased by 50\% & \\
\hline & & - PEF readings increased by $11 \%$ & \\
\hline & & - Reduction in days off work & \\
\hline & & - Reduction of inhaled beta-agonists by $50 \%$ & \\
\hline & & - Overall quality of life improved by $19 \%$ & \\
\hline & & $\begin{array}{l}\text { - Emergency room visits and medical visits } \\
\text { decreased by } 75 \%\end{array}$ & \\
\hline & Van der $\mathrm{Meer}^{33}$ & - Cost increase of 641 USD per patient & - 26,700 USD per QALY gained \\
\hline & & & \\
\hline & Schermer ${ }^{41}$ (2002) & $\begin{array}{l}\text { - Cost savings of } 13 \text { Euro per patient } \\
\text { - QALY gain of } 0.039 \text { per patient }\end{array}$ & - 33 Euro per successfully treated/week \\
\hline \multirow[t]{12}{*}{ Diabetes } & Gillet $^{24}(2010)$ & - Cost increase of $209 \mathrm{GBP}$ (trial based costs) and $82 \mathrm{GBP}$ & - 5,387 GBP per QALY (trial based) \\
\hline & & $\begin{array}{l}\text { ('real life' costs) per patient } \\
\text { - QALY gain of } 0.0392 \text { per patient }\end{array}$ & - 2,092 GBP per QALY ('real life' costs) \\
\hline & Handley $^{25}$ (2008) & - Cost increase of 782 USD per patient & - 65,167 USD per QALY gained \\
\hline & & - QALY gain of 0.012 per patient & \\
\hline & $\operatorname{Jansa}^{26}(2006)$ & - Cost savings of 275 USD per patient & $\mathrm{NI}$ \\
\hline & & - No differences in HbAlc & \\
\hline & & - 9\% less patients with hypoglycemic events & \\
\hline & & - Points improvement DQOL & \\
\hline & & - Point decrease SF-12 & \\
\hline & & - Point decrease diabetes knowledge test & \\
\hline & & - 12\% less patients with glycemic controls & \\
\hline & Ritzwoller ${ }^{39}$ (2011) & $\begin{array}{l}\text { - Self-management: 4,634 USD total costs per patient } \\
\text { and 7,723 USD marginal costs }\end{array}$ & $\mathrm{Nl}$ \\
\hline \multirow{7}{*}{$\begin{array}{l}\text { Cardiovascular } \\
\text { disease }\end{array}$} & Cline $^{22}(1998)$ & - Cost saving of 1,300 USD per patient & $\mathrm{Nl}$ \\
\hline & & - One year survival rate $1.8 \%$ higher for SMI & \\
\hline & Jowett ${ }^{27}(2006)$ & - Cost increase of $295 \mathrm{GBP}$ per patient & - 31,437 GBP per QALY gained \\
\hline & & - No significant differences in QALYS & \\
\hline & $\operatorname{Reed}^{37}(2010)$ & - Cost increase of 297 USD per patient & - 11,570 USD per life-year saved \\
\hline & & - Improvement of blood pressure control of $11 \%$ & \\
\hline & & - Other outcomes no significant differences & \\
\hline
\end{tabular}


van Eeden et al.

Table 3. Continued

\begin{tabular}{|c|c|c|c|}
\hline Disease & Author & Main outcome ${ }^{a}$ & ICER \\
\hline \multirow[t]{2}{*}{ COPD } & Bourbeau $^{21}(2006)$ & $\begin{array}{l}\text { - Cost savings of 2,149 USD per patient } \\
\text { - Reduced hospital admissions 39.8\% } \\
\text { - Reduced emergency department visits 41\% } \\
\text { - Reduced unscheduled physician visits } 58.9 \%\end{array}$ & $\begin{array}{l}-4,214 \text { USD }(n=14), 2,053 \text { USD }(n=30) \\
1,326 \text { USD }(n=50) \text { and } 1,016 \text { USD }(n=70) \\
\text { per hospitalisation prevented }\end{array}$ \\
\hline & Khdour $^{30}(2011)$ & $\begin{array}{l}\text { - Cost savings of } 672 \text { GBP per patient } \\
\text { - QALY gain of } 0.065 \text { per patient }\end{array}$ & - 3,278 GBP per QALY gained \\
\hline \multirow[t]{2}{*}{$\begin{array}{l}\text { Neurological } \\
\text { problems }\end{array}$} & Latimer $^{31}(2013)$ & $\begin{array}{l}\text { - SMI: 19,124 GBP and 3.22 QALY gain per person } \\
\text { - Usual care: 19,687 GBP and 3.07 QALY gain per person } \\
\text { - Incremental costs and incremental QALY: } 437 \text { GBP and 0.14 }\end{array}$ & - 3,058 GBP per QALY gained \\
\hline & $\begin{array}{l}\text { Moss-Morris }^{34} \\
\quad(2012)\end{array}$ & $\begin{array}{l}\text { - SMl: } 211 \text { GBP mean costs and 0.12 QALY gain } \\
\text { - Usual care: } 214 \text { GBP mean costs and 0.12 QALY gain } \\
\text { - Due to higher baseline QALY for intervention group, } \\
\text { QALY gain for intervention group was higher }\end{array}$ & $\mathrm{Nl}$ \\
\hline \multirow[t]{6}{*}{ Other } & $\begin{array}{l}\text { Van Os-Medendorp }{ }^{35} \\
\quad(2012)\end{array}$ & $\begin{array}{l}\text { - E-health self-management training would save } 594 \text { USD per } \\
\text { patient in the first year after treatment } \\
\text { - E-health is } 73 \% \text { likely to be cost-effective }\end{array}$ & $\mathrm{Nl}$ \\
\hline & Patel 36 (2009) & $\begin{array}{l}\text { - Cost increase of } 101 \mathrm{GBP} \text { per patient } \\
\text { - No significant differences in QALYs between interventions }\end{array}$ & $\begin{array}{l}\text { - } 279 \text { GBP per QALY gained (SF-36 mental health } \\
\text { component) } \\
\text { - 1,189 GBP per QALY gained (SF-36 physical } \\
\text { health component) } \\
\text { - 13,473 GBP per QALY gained (VAS) }\end{array}$ \\
\hline & Richardson $^{38}$ (2013) & $\begin{array}{l}\text { - SMI: }+218 \text { GBP and - }-012 \text { QALY vs. care as usual } \\
\text { - Supportive listening: }+460 \text { GBP and -.042 QALY vs care as } \\
\text { usual } \\
\text { - Care as usual is dominant vs. both treatments, } \\
65 \% \text { likelihood of being cost effective }\end{array}$ & $\mathrm{Nl}$ \\
\hline & Robinson ${ }^{40}(2005)$ & $\begin{array}{l}\text { - Cost savings of } 73 \text { GBP (or } 40 \% \text { ) } \\
\text { - Primary care consultations reduced with 60\% }\end{array}$ & $\mathrm{Nl}$ \\
\hline & Strong ${ }^{42}(2006)$ & $\begin{array}{l}\text { - Cost increase of } 168 \text { USD and } 172 \text { USD } \\
\text { - Low-impact back pain days increase with } 6 \text { and } 24\end{array}$ & $\begin{array}{l}\text { - } 9.70 \text { USD per low back-pain day (lay intervention) } \\
\text { - 6.13 USD per low back-pain day } \\
\text { (psychologist intervention) }\end{array}$ \\
\hline & Wang $^{43}$ (2012) & $\begin{array}{l}\text { - No significant changes in costs and effects after } \\
18 \text { months follow-up }\end{array}$ & $\mathrm{Nl}$ \\
\hline
\end{tabular}

ICER, incremental cost-effectiveness ratio; OK, Norwegian Krone; SGRQ, St. George's Respiratory Questionnaire; REV, forced respiratory volume; SMI, self-management intervention; FIM, Finnish Markka; 15D, Health State Descriptive System Questionnaire; FVC, forced vital captivity; PD, airway hyper responsiveness; PEF, peak expiratory flow; QALY, quality-adjusted life year; SF-36, short-form 36.

cost-effective. However, the cost-effectiveness results of the studies included in this review must be interpreted with caution because thirteen studies with a moderate or low quality score conclude that the SMI is cost-effective. It is imperative that these conclusions are preceded by a solid methodological study quality on the one hand and a clear and explicit understanding of the theoretical context of the SMI on the other hand.

In this review, we explored the methodological quality of economic evaluation studies of SMIs in adult chronic pa- tients, and investigated their cost-effectiveness. Our findings were in line with conclusions from previous related research. Willems et al. (9) conducted a review on the cost-effectiveness of SMIs in asthma and concluded that due to wide diversity in interventions and poor methodological quality, conclusions had to be interpreted with caution. Dorn (46) concluded that self-management has the potential to improve outcomes for chronic patients; however, the quality of studies was subpar due to the challenge of defining self-management and developing 
practical self-management interventions. In addition to previous findings, we find it remarkable that the increased popularity of SMIs has not been accompanied by the development of a clear, well-considered definition of the concept.

\section{Limitations of This Review}

First, the difficulties we encountered with the concept of self-management can be considered a limitation, as already mentioned in previous parts of the discussion. However, we chose a well-known definition of self-management after reviewing relevant literature, and two independent assessors decided whether the SMI was in line with our chosen definition of selfmanagement. Second, we did not register this systematic review at PROSPERO but we did use a predefined research protocol. Third, we chose 1990 as the starting year for our search strategy, so we may have missed relevant articles published before this year. However, because the domain of both economic evaluation and self-management have been subject to scientific research mainly only for the past two decades (only two studies included in this review were published before 2000), we do not expect to have missed many relevant publications. Fourth, we used only two databases for our search strategy. Because NHSEED systematically searches multiple databases, all 23 studies included in this review can be found in both PUBMED and NHSEED, and a reference check of all included studies did not result in additional relevant studies we can argue that these two databases suffice for this review. Fifth, conference abstracts were excluded; hence, we could have missed some important on-going studies. However, because it would not be possible to analyze these data with the chosen research method we decided to exclude them. Finally, the use of the adjusted version of the CHEC-list may count as a limitation. The questions for the model-based studies were not valued by a Delphi panel yet (19). However, as only one of the included studies in our review was a model-based economic evaluation this will not have influenced our study findings.

\section{CONCLUSION}

From this systematic review, we can conclude that the SMIs of adult chronic patients reported were heterogeneous and no clear, well-considered definition of self-management was used. Furthermore, the number of economic evaluation studies of SMIs is increasing, as is methodological quality. However, in general the methodological quality of the assessed full economic evaluation studies was moderate, the cost-effectiveness results must, therefore, be interpreted with caution.

\section{RECOMMENDATIONS}

For future research, we recommend that the methodological quality of economic evaluation studies of SMIs must be improved. One of the major difficulties we encountered was with the taxonomy of self-management and SMIs. We strongly rec- ommend that future research efforts focus on defining the concept of self-management in such a way that it diminishes the fogginess around this concept. Not only researchers, but also healthcare decision makers, professionals and patients will benefit of this. Other difficulties related to the methodological quality of the economic evaluation studies. Up to date methods must be used for conducting economic evaluations such as the CHEERS guidelines (47). Furthermore, more effort must be put in improving trial design and reporting. We would advise that future research uses the adjusted CHEC-list (19) to assess modelbased studies and to develop for CHEC-list items. In addition, we found a very limited number of economic evaluation studies in comparison with effect studies on self-management, which stresses the importance of conducting more economic evaluation studies attached to effect studies on self-management.

\section{SUPPLEMENTARY MATERIAL}

Supplementary Figure 1

Supplementary Tables 1 and 2

http://dx.doi.org/10.1017/S0266462316000027

\section{CONFLICTS OF INTEREST}

The authors declare that they have no competing interests.

\section{REFERENCES}

1. World Health Organization. Health topics. Geneva: WHO; 2012.

2. Barlow J, Wright C, Sheasby J, Turner A, Hainsworth J. Self-management approaches for people with chronic conditions: A review. Patient Educ Couns. 2002;48:177-187.

3. Suhrcke M, Nugent R, Stuckler D, Rocco L. Chronic disease: An economic perspective. The Oxford Health Alliance. 2006:60.

4. Lorig KR, Sobel DS, Stewart AL, et al. Evidence suggesting that a chronic disease self-management program can improve health status while reducing hospitalization: A randomized trial. Med Care. 1999;37:5-14.

5. Bandura A. Self-efficacy: Toward a unifying theory of behavioral change. Psychol Rev. 1977;84:191-215.

6. Franek J. Self-management support interventions for persons with chronic disease: An evidence-based analysis. Ont Health Technol Assess Ser. 2013;13:1-60.

7. Janson SL, McGrath KW, Covington JK, Cheng SC, Boushey HA. Individualized asthma self-management improves medication adherence and markers of asthma control. $j$ Allergy Clin Immunol. 2009;123:840-846.

8. Warsi A, Wang PS, LaValley MP, Avorn J, Solomon DH. Selfmanagement education programs in chronic disease: A systematic review and methodological critique of the literature. Arch Intern Med. 2004;164:1641-1649

9. Willems DC, Joore MA, Hendriks JJ, Wouters EF, Severens JL. Costeffectiveness of self-management in asthma: A systematic review of peak flow monitoring interventions. Int $J$ Technol Assess Health Care. 2006;22:436-442.

10. Drummond MF, Jefferson TO. Guidelines for authors and peer reviewers of economic submissions to the bmj. The bmj economic evaluation working party. BMJ. 1996;313:275-283.

11. Martin CM. Chronic disease and illness care: Adding principles of family medicine to address ongoing health system redesign. Can Fam Physician. 2007;53:2086-2091. 
12. Alton V, Eckerlund I, Norlund A. Health economic evaluations: How to find them. Int J Technol Assess Health Care. 2006;22:512-517.

13. Sassi F, Archard L, McDaid D. Searching literature databases for health care economic evaluations: How systematic can we afford to be? Med Care. 2002;40:387-394.

14. Gibson PG, Powell H, Coughlan J, et al. Self-management education and regular practitioner review for adults with asthma. Cochrane Database Syst Rev. 2003:CD001117.

15. Jones F, Riazi A. Self-efficacy and self-management after stroke: A systematic review. Disabil Rehabil. 2011;33:797-810.

16. Newman S, Steed L, Mulligan K. Self-management interventions for chronic illness. Lancet. 2004;364:1523-1537.

17. Evers SM, Van Wijk AS, Ament AJ. Economic evaluation of mental health care interventions. A review. Health Econ. 1997;6:161-177.

18. Higgins JPT, Green S (editors). Cochrane handbook for systematic reviews of interventions. Version 5.0.2 [updated September 2009]. The Cochrane Collaboration; 2009. Available from www. cochrane-handbook.org.

19. Odnoletkova I, Goderis G, Lore P, et al. Systematic review on the costeffectiveness of therapeutic education to prevent the development and progression of type 2 diabetes. Value Health. 2013;16:452.

20. Evers S, Goossens M, de Vet H, van Tulder M, Ament A. Criteria list for assessment of methodological quality of economic evaluations: Consensus on health economic criteria. Int J Technol Assess Health Care. 2005;21:240-245.

21. Bourbeau J, Collet J-P, Schwartzman K, et al. Economic benefits of self-management education in COPD. Chest. 2006;130:1704-1711.

22. Cline C, Israelsson B, Willenheimer R, Broms K, Erhardt L. Cost effective management programme for heart failure reduces hospitalisation. Heart. 1998;80:442-446.

23. Gallefoss F, Bakke P. Cost-effectiveness of self-management in asthmatics: A 1-yr follow-up randomized, controlled trial. Eur Respir J. 2001;17:206-213.

24. Gillett M, Dallosso H, Dixon S, et al. Delivering the diabetes education and self management for ongoing and newly diagnosed (desmond) programme for people with newly diagnosed type 2 diabetes: Cost effectiveness analysis. BMJ. 2010;341:c4093.

25. Handley MA, Shumway M, Schillinger D. Cost-effectiveness of automated telephone self-management support with nurse care management among patients with diabetes. Ann Fam Med. 2008;6:512-518.

26. Jansa M, Vidal M, Viaplana J, et al. Telecare in a structured therapeutic education programme addressed to patients with type 1 diabetes and poor metabolic control. Diabetes Res Clin Pract. 2006;74:26-32.

27. Jowett S, Bryan S, Murray E, et al. Patient self-management of anticoagulation therapy: A trial-based cost-effectiveness analysis. Br J Haematol. 2006; 134:632-639.

28. Kauppinen R, Sintonen H, Tukiainen H. One-year economic evaluation of intensive vs conventional patient education and supervision for selfmanagement of new asthmatic patients. Respir Med. 1998;92:300-307.

29. Kauppinen R, Vilkka V, Sintonen H, Klaukka T, Tukiainen H. Longterm economic evaluation of intensive patient education during the first treatment year in newly diagnosed adult asthma. Respir Med. 2001;95:5663.

30. Khdour MR, Agus AM, Kidney JC, et al. Cost-utility analysis of a pharmacy-led self-management programme for patients with COPD. Int J Clin Pharm. 2011;33:665-673.
31. Latimer NR, Dixon S, Palmer R. Cost-utility of self-managed computer therapy for people with aphasia. Int J Technol Assess Health Care. 2013;29:402-409.

32. McLean W, Gillis J, Waller R. The bc community pharmacy asthma study: A study of clinical, economic and holistic outcomes influenced by an asthma care protocol provided by specially trained community pharmacists in british columbia. Can Respir J. 2003;10:195202.

33. van der Meer V, van den Hout WB, Bakker MJ, et al. Cost-effectiveness of internet-based self-management compared with usual care in asthma. PLoS One. 2011;6:e27108.

34. Moss-Morris R, McCrone P, Yardley L, et al. A pilot randomised controlled trial of an internet-based cognitive behavioural therapy selfmanagement programme (ms invigor8) for multiple sclerosis fatigue. Behav Res Ther. 2012;50:415-421.

35. van Os-Medendorp H, Koffijberg H, Eland-de Kok PC, et al. E-health in caring for patients with atopic dermatitis: A randomized controlled cost-effectiveness study of internet-guided monitoring and online selfmanagement training. Br J Dermatol. 2012;166:1060-1068.

36. Patel A, Buszewicz M, Beecham J, et al. Economic evaluation of arthritis self management in primary care. BMJ. 2009;339:b3532.

37. Reed SD, Li Y, Oddone EZ, et al. Economic evaluation of home blood pressure monitoring with or without telephonic behavioral self-management in patients with hypertension. Am $J$ Hypertens. 2010;23:142-148.

38. Richardson G, Epstein D, Chew-Graham C, et al. Cost-effectiveness of supported self-management for $\mathrm{cfs} / \mathrm{me}$ patients in primary care. $B M C$ Fam Pract. 2013;14:12.

39. Ritzwoller DP, Sukhanova AS, Glasgow RE, et al. Intervention costs and cost-effectiveness for a multiple-risk-factor diabetes self-management trial for Latinas: Economic analysis of viva bien! Transl Behav Med. 2011;1:427-435.

40. Robinson A, Lee V, Kennedy A, et al. A randomised controlled trial of self-help interventions in patients with a primary care diagnosis of irritable bowel syndrome. Gut. 2006;55:643-648.

41. Schermer TR, Thoonen BP, van den Boom G, et al. Randomized controlled economic evaluation of asthma self-management in primary health care. Am J Respir Crit Care Med. 2002;166:1062-1072.

42. Strong LL, Von Korff M, Saunders K, Moore JE. Cost-effectiveness of two self-care interventions to reduce disability associated with back pain. Spine (Phila Pa 1976). 2006;31:1639-1645.

43. Wang V, Smith VA, Bosworth HB, et al. Economic evaluation of telephone self-management interventions for blood pressure control. Am Heart J. 2012;163:980-986.

44. Hurley MV, Walsh NE, Mitchell HL, et al. Economic evaluation of a rehabilitation program integrating exercise, self-management, and active coping strategies for chronic knee pain. Arthritis Rheum. 2007;57:12201229.

45. Nolte S, Osborne RH. A systematic review of outcomes of chronic disease self-management interventions. Qual Life Res. 2012;22:18051816.

46. Dorn S. Systematic review: Self-management support interventions for irritable bowel syndrome. Aliment Pharmacol Ther. 2010;32:513-521.

47. Husereau D, Drummond M, Petrou S, et al. Consolidated health economic evaluation reporting standards (CHEERS) statement. Int J Technol Assess Health Care. 2013;29:117-122. 